\title{
The discoid meniscus
}

\author{
Moshe Yaniv $\cdot$ Nehemia Blumberg
}

Received: 10 April 2007/ Accepted: 14 May 2007/Published online: 26 June 2007

(C) EPOS 2007

\begin{abstract}
Discoid lateral meniscus is an intra-articular knee disorder typically presented in the young population and during adolescence. Different types of meniscal disorders and varied forms of presentation have been reported. The natural history depends on the type of anomaly and the presence of symptoms. Management of the disorder should be directed toward the resolution of the symptoms while preserving meniscal tissue and function. Modern surgical techniques enable suturing and preservation of meniscal tissue. The clinical manifestations, diagnostic modalities and criteria, accompanying conditions and practical management considerations are reviewed.
\end{abstract}

Keywords Discoid lateral meniscus - Adolescence · Meniscal tear . "Snapping knee"

\section{Introduction}

Discoid lateral and medial menisci were first described in cadaver specimens by Young in 1889 [1] and WatsonJones in 1930 [2], respectively. Descriptions of the anatomy, development and clinical manifestation of this anomaly soon followed, classifications of its various forms

\section{Yaniv ( $\varangle)$}

Department of Pediatric Orthopaedics, Dana Children's

Hospital, Tel Aviv Sourasky Medical Center, 6 Weizman Street,

Tel Aviv 64239, Israel

e-mail: pedorth@tasmc.health.gov.il; yaniv@ sportoped.com

N. Blumberg

Department of Orthopedic Surgery "B", Tel Aviv

Sourasky Medical Center, Sackler Faculty of Medicine,

Tel Aviv University, Tel Aviv, Israel were proposed, and treatment protocols were established. This review describes the origin and nature of the pathology and outlines the current knowledge of this knee disorder.

\section{Embryology and anatomy}

The normal menisci differentiate within the limb bud from mesenchymal tissue early during fetal development. They are clearly defined at the 8th week of gestation and gain mature anatomical shape at the 14th week [3], without ever possessing a discoid shape [4]. Their peripheral blood supply recedes during maturity. By 9 months of life, the central third becomes avascular, and only the peripheral third retains its blood supply at adulthood (approximately 10 years of age) [5] - the inner two-thirds receive nourishment via diffusion from the intra-articular fluid.

In adults, the C-shaped medial meniscus covers $50 \%$ of the medial tibial plateau and is connected firmly to the joint capsule (coronary, meniscotibial and deep medial collateral ligament). The average lateral meniscus is $12 \mathrm{~mm}$ wide, $4 \mathrm{~mm}$ high and circular in shape, although the normal anatomy varies considerably with regard to dimension and shape [6]. It covers $70 \%$ of the lateral tibial plateau and has firm anterior and posterior attachments (augmented posteriorly by the anterior and posterior meniscofemoral ligaments), while its lateral joint capsule attachment is loose (i.e., there is no attachment at the popliteal hiatus and lateral collateral ligament). Thus, the average excursion of the meniscus during flexion and extension is greater laterally $(10 \mathrm{~mm}$ vs. $2.5 \mathrm{~mm})$, a feature that protects against the incidence of lateral meniscal tear [7]. The flat tibial and concave femoral meniscal surfaces cushion the tibial plateau and femoral condyle, respectively. 


\section{Incidence}

The actual incidence of discoid menisci is difficult to estimate due to the high rate of asymptomatic patients. The reported incidence of discoid meniscus ranges from $0.4 \%$ to $17 \%$ for the lateral $[7,8]$ and $0.06 \%$ to $0.3 \%$ for the medial [9-11] side. The condition is more frequently reported in Asian countries [6, 12-14]. The incidence of bilateral lateral discoid meniscus is up to $20 \%$ of the cases, whereas bilateral medial involvement is rare [11].

\section{Etiology}

There are several theories of the etiology of discoid meniscus. In 1948, Smillie [15] postulated that the discoid shape is a normal stage in the developing embryo and that failure of absorption of the central portion will persist during the fetal state ("congenital discoid meniscus"). His theory was later refuted because neither human nor animal embryological specimens demonstrated such a stage during normal development [4, 5]. The "developmental" theory, proposed by Kaplan [4], claimed that insufficient posterior meniscal attachment to the tibia causes increased meniscal excursion during flexion and extension and, so, repetitive microtrauma subsequent to this increased mobility produces morphological changes. Kaplan's theory, however, does not explain the majority of discoid meniscus presentations, which have normal posterior attachment. The "congenital" theory, which is supported by case reports of the occurrence of the condition in twins [16] and descriptions of familial transmission [17], considers the discoid meniscus as an anatomical variant and suggests that increased shear stress causes meniscocapsular separation and secondary hypermobility.

\section{Classifications}

Watanabe et al. [18] classified various types of lateral discoid meniscus based on its arthroscopic appearance. Discoid menisci with normal peripheral attachments were labeled as either type I (complete) or type II (incomplete) according to the degree of coverage of the lateral tibial plateau. Type III (also the Wrisberg ligament type) includes cases without normal posterior meniscotibial attachment, thus allowing increased mobility and producing the classic "snapping knee" syndrome. Although this is the most frequently used classification system, its value for the purposes of treatment decision-making is somewhat questionable. The traditional classification was expanded by Monllau et al. [19] who added a fourth type to describe a ring-shaped meniscus characterized by a ring-shaped morphology with a normal posterior tibial attachment.
Bin et al. [20] modified the well-known O'Connor [21] general intraoperative classification of meniscal tears and described them as simple horizontal, complicated horizontal, longitudinal, radial, degenerative, and complex. They found a relationship between the amount of surgical resection to stable margins and to the tear pattern, but not to the type of discoid according to Watanabe et al. [18]. Only partial meniscectomy needed to be carried out in radial and degenerative tears, whereas subtotal or total meniscectomy was required significantly more frequently in longitudinal and complex tears. They concluded that their method was useful for treatment planning.

Jordan et al. [6, 14] based their classification on both clinical and intraoperative findings. They defined the meniscal type (complete or incomplete), its peripheral rim stability, and the presence or lack of symptoms and tear.

\section{Clinical presentation}

The term "snapping knee syndrome" was attributed to discoid meniscus by Kroiss in 1910 [22] because of its clinical presentation. The symptoms, however, are actually highly variable, depending on the type of the discoid meniscus, its location (medial or lateral), the presence of tear, and the status of rim stability [6, 13, 23-27].

Stable discoid meniscus is often an incidental finding in asymptomatic patients. It can become symptomatic in the presence of a tear but, unlike a normally shaped meniscus, the onset of symptoms can be insidious without an acute trauma. The most common tear pattern is that of degenerative horizontal cleavage, which comprises 58-98\% of all cases of symptomatic discoid meniscus [28-30].

Unstable discoid meniscus produces the classical "snapping knee". It is usually related to the less common Wrisberg ligament type, but it can also be seen when a torn stable discoid meniscus becomes unstable due to spread of a tear that includes the posterior tibial attachment. An audible palpable or visible snap on terminal extension $\left(10-20^{\circ}\right)$ along with pain swelling and locking, in the absence of a traumatic cause, are the chief presentations of young children with this syndrome. On physical examination, the patient may present with effusion, limited extension, anterolateral bulge at full flexion, pain, and/or joint line tenderness. A positive McMurray test may be found, but it is not typical especially in young children. A true locking is a rare type of presentation; pseudo-locking may occur, but requires no specific maneuver to restore range of motion. The overall accuracy of clinical examination ranges from $29 \%$ to $93 \%$ when compared with knee arthroscopy findings-being dependent on the examiner's experience in dealing with sports injuries of children and young adolescents [31-36]. The variation in symptoms, their descriptions 

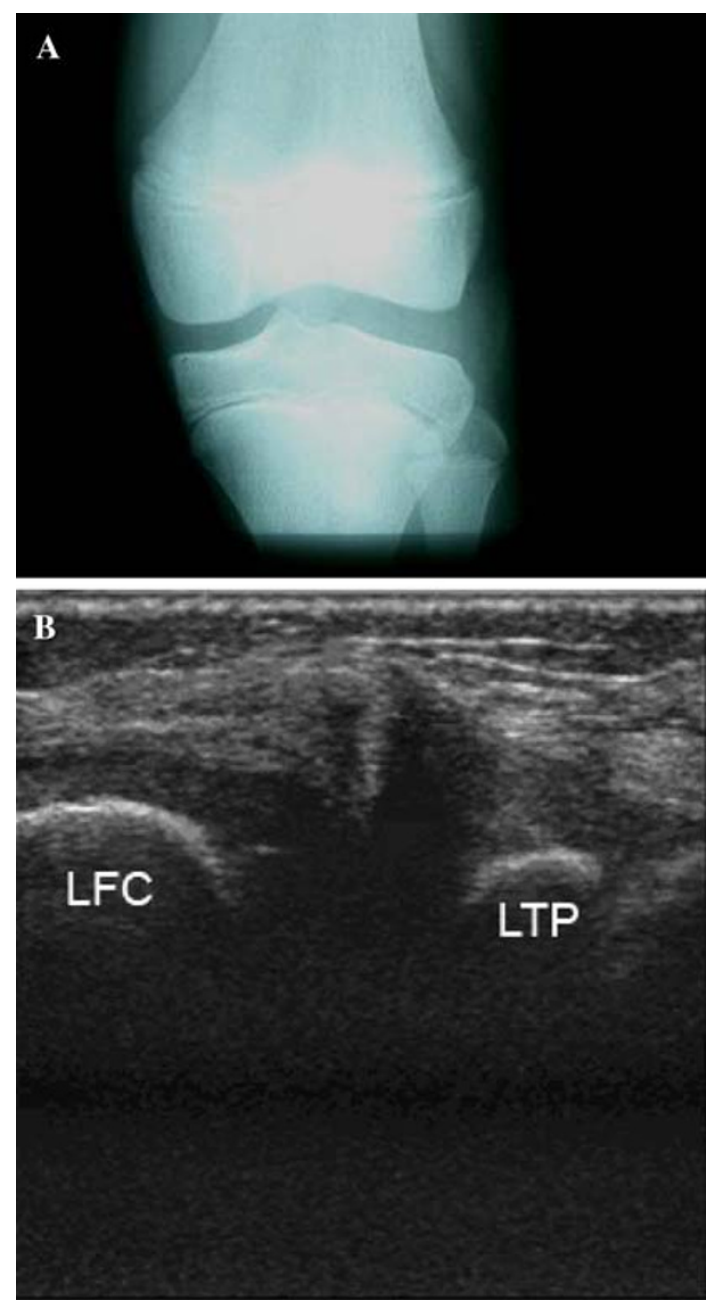

Fig. 1 a Plain X-ray (anterior-posterior view) of a 10-year-old boy's knee demonstrating widening of the lateral joint space, squaring of the lateral femoral condyle, cupping of the lateral tibial plateau, and tibial eminence hypoplasia. b An ultrasound of the wide and irregular lateral meniscus ( $L F C$ lateral femoral condyle, LTP lateral tibial plateau)

as being intermittent and vague, an insidious onset, and difficulty in precise anamnesis and physical examination all contribute to inconsistencies in the clinical examination, thus necessitating further workup, such as imaging studies.

\section{Imaging}

\section{Radiography}

According to Picard and Constantin's 1964 publication [37], standard anterior-posterior, lateral, tunnel, and skyline views contribute significantly to the establishment of diagnosis. Lateral joint space narrowing, squaring of the lateral femoral condyle, cupping of the lateral tibial plateau, tibial eminence hypoplasia, and fibular head elevation may be demonstrated [38]. These radiographic findings-especially as an entire set-are present only in some cases (Fig. 1a). Associated pathologies affecting the lateral femoral condyle, such as osteochondritis dissecans, may also be visualized.

\section{Ultrasonography}

Ultrasonographic imaging of the menisci may demonstrate a wide and irregularly shaped lateral discoid meniscus in type 1 and 2 discoid menisci (Fig. 1b). Due to its availability, multiplanar capability, and economic benefit, sonography has been used to evaluate meniscal tears in the knee, with an overall accuracy of more than $70 \%$. The use of high-resolution micro convex probes, which better fit the anatomic concavity of the popliteal fossa, achieves a sensitivity of $100 \%$ and a specificity of $95 \%$ in detecting meniscal tears. The positive predictive value was reported to be $95 \%$ and $93 \%$ for the medial and lateral menisci, respectively, and the negative predictive value was $100 \%$ [39]. It is important to point out that ultrasonography (US) is an examiner-dependent tool and should be evaluated accordingly in the clinical setting. Achour et al. [40] recently reported on eight children (aged 6-11 years) who had been investigated by means of US for clinically suspected meniscal pathology. The sonographic criteria for diagnosis of discoid meniscus were the absence of a normal triangular shape, the presence of an abnormally elongated and thick meniscal tissue, and the demonstration of a heterogeneous central pattern. Associated tears and degeneration were well demonstrated on US, and arthroscopy confirmed the sonographic findings in all cases. The authors concluded that US is a reliable technique for the diagnosis of discoid meniscus in children.

\section{Magnetic resonance imaging}

Magnetic resonance imaging (MRI) is widely used to diagnose musculoskeletal pathologies affecting the knee since it gives a clear picture of soft-tissue structures. The most accurate criteria for the diagnosis of discoid meniscus on MRI are a ratio of the minimal meniscal width to maximal tibial width (on the coronal slice) of more than $20 \%$ and a ratio of the sum of the width of both lateral horns to the meniscal diameter (on the sagittal slice showing the maximal meniscal diameter) of more than $75 \%$. Both ratios had a sensitivity and specificity of $95 \%$ and $97 \%$, respectively, even when torn menisci were present [41]. Other less precise criteria were a minimal meniscal width (on the coronal slice) of more than $15 \mathrm{~mm}$, and three or more consecutive sagittal slices showing continuity between the anterior and posterior horns of the meniscus. A significant difference in the sensitivity of 

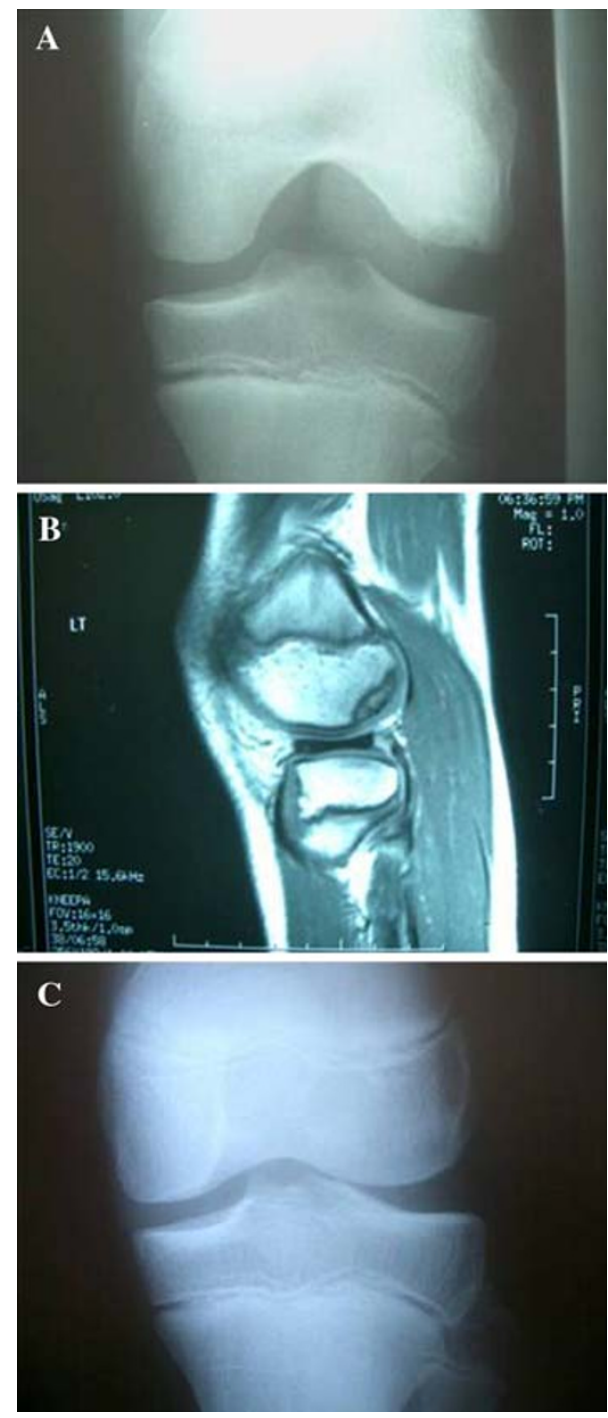

Fig. 2 a Tunnel view of the left knee demonstrating signs of lateral discoid meniscus accompanied by an osteochondral lesion of the lateral femoral condyle. b Magnetic resonance imaging: a sagittal view demonstrating the same lesion. c Same knee 6 months after reshaping of the lateral discoid meniscus (note resolution of the osteochondral lesion)

lateral discoid meniscus diagnosis, between clinical examination and MRI study (clinical examination, 88.9\%; MRI study $38.9 \%$ ) was reported in the pediatric population [31].

MRI can also provide information on intra-substance tissue, meniscal tear, and the presence of associated osteochondritis dissecans. Incomplete, Wrisberg ligament type or unstable, normally shaped, menisci are much more difficult to discern [42]. A ring-shaped meniscus is not easily distinguished from a bucket handle tear of the normal lateral meniscus using MRI [43]. Improved MR sequences for better sensitivity may include arthrography.

\section{Accompanying conditions}

Discoid lateral meniscus was reported to be associated with other musculoskeletal anomalies, among them high fibular head, fibular muscular defects, hypoplasia of the lateral femoral condyle with lateral joint-space widening, hypoplasia of the lateral tibial spine, abnormally shaped lateral malleolus of the ankle, and enlarged inferior lateral geniculate artery.

One of the most clinically demanding conditions is the association between lateral discoid meniscus and an osteochondral lesion of the lateral femoral condyle (Fig. 2a-c).

Osteochondritis dissecans of the lateral femoral condyle is relatively rare and is reported to be often combined with lateral discoid meniscus. The presence of lateral discoid meniscus was reported to occur in a majority (80-100\%) of the osteochondritis dissecans lesions that occurred in the lateral femoral condyle [44]. It was suggested that existence of a discoid meniscus itself might produce an abnormal contact force onto the lateral femoral condyle even if the meniscus is not torn. This abnormal contact force may lead to an osteochondritis dissecans lesion in the lateral femoral condyle [45]. Based on clinical parameters and knee alignment measurements, Terashima et al. [46] concluded that a lateral discoid meniscus tear, young age and high activity, and valgus alignment were predisposing factors for osteochondritis dissecans of the lateral femoral condyle. Meniscoplasty was shown to permit the healing of an osteochondral lesion and it was the recommended approach for osteochondritis dissecans of the lateral femoral condyle when combined with the lateral discoid meniscus [44].

Development of juvenile osteochondritis dissecans (JOCD) following discoid lateral meniscal sculpturing or total lateral meniscectomy was reported [47, 48]. Changes of the load transmission profile in the lateral compartment was considered as the etiology of these findings.

\section{Treatment}

Traditionally, the treatment of choice for symptomatic stable or unstable discoid lateral meniscus was open total meniscectomy. The residual meniscal tissue that had been left after partial meniscectomy was considered abnormal and was, therefore, supposed to be resected as well [9, 15, 24, 25]. The menisci serve as load distributors and shock absorbers and play a role in joint stability as well as in synovial fluid distribution and cartilage nutrition. Better understanding and documentation of the importance of the menisci to normal articular function has led to preservation of stable meniscal tissue as part of treatment planning. 

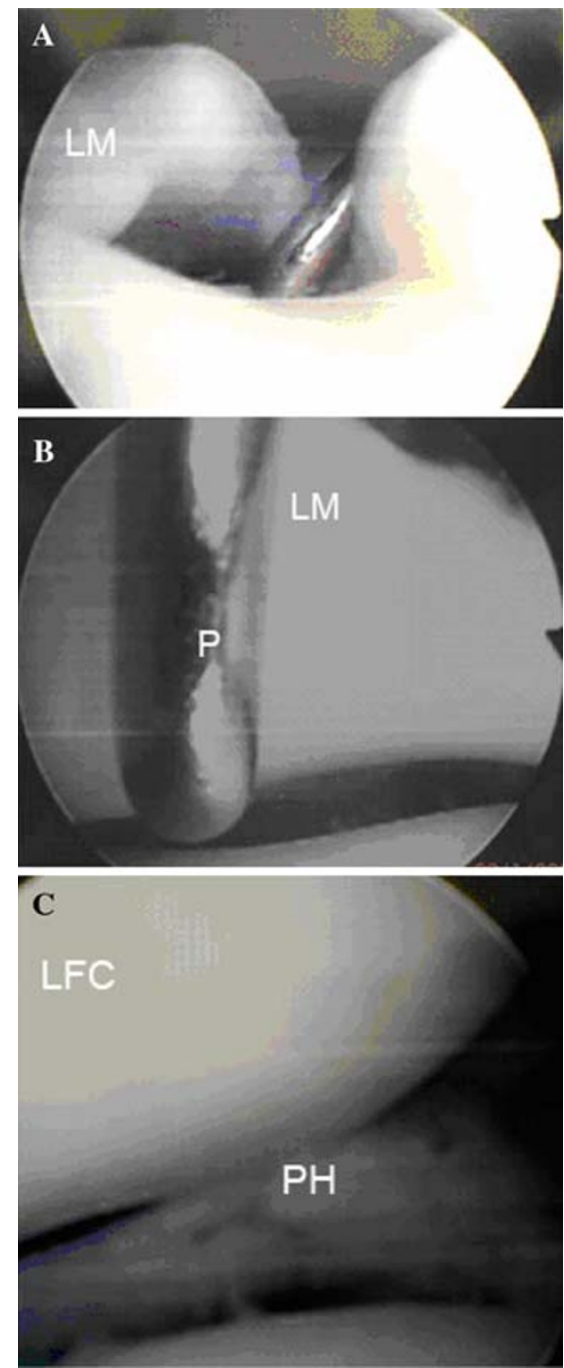

Fig. 3 Arthroscopic demonstration of complete lateral discoid meniscus. a Wide medial edge of the lateral meniscus. The arthroscopic probe points the small tibial plateau part not covered with the meniscus ( $L M$ lateral meniscus). b Demonstration of the wideness architecture of the discoid meniscus inner edge, more than the 3-mm long arthroscopic probe ( $L M$ lateral meniscus, $P$ Probe). c Same meniscus after reshaping and suturing of the posterior unstable horn ( $L F C$ lateral femoral condyle, $P H$ posterior horn lateral meniscus, $T P$ tibial plateau)

Partial meniscectomy of normal shaped menisci, was shown to increase the contact stresses in proportion to the amount of removed meniscus [49]. Following total meniscectomy, the contact area was decreased by $75 \%$ while contact stresses increased by $235 \%$ [49]. The lack of normal meniscal fibrocartilage arrangement in discoid menisci types 1 and 2 may play a roll in load changes after partial meniscectomy of lateral discoid meniscus, but no data supporting it has yet been published. A meniscus-deficient knee carries a high risk of early cartilage degeneration and early degenerative changes. Total meniscectomy of a non-discoid meniscus often leads to osteoarthritis [50-54]. Bearing in mind the detrimental effect of meniscectomy on the knee's function, the goal in treatment planning should be preservation of meniscal tissue.

Incidentally found discoid lateral meniscus with no symptoms or physical signs should not be treated surgically. A periodic follow-up for exclusion of symptoms and a physical examination enables early detection of any deterioration and appropriate treatment planning.

Snapping knee with no other symptoms and no radiographic signs of accompanying articular lesions can be followed-up and then treated should it become symptomatic. A patient may become symptomatic due to instability of the meniscus, a new tear of the ill-defined meniscus, or as the result of accompanying findings, such as osteochondral lesions to the lateral joint compartment.

Arthroscopic partial meniscectomy (saucerization) is the treatment of choice for symptomatic stable, complete, or incomplete discoid lateral meniscus $[8,30,55]$. The width of the remaining peripheral rim is an important feature to consider when meniscectomy is performed. Most authors agree that the width of the remaining peripheral rim should be between $5 \mathrm{~mm}$ and $8 \mathrm{~mm}$ to prevent impingement and instability of the remaining part of a discoid lateral meniscus that may lead to future secondary meniscal tear $[55,56]$. If a meniscal tear is present, partial central meniscectomy in conjunction with suture repair of the peripheral tear can be effective treatment [57] (Fig. 3a-c).

The reparability of the lateral discoid meniscus cannot be reliably predicted from imaging studies, even MRI [58], and can best be decided on intraoperatively. Klingele et al. [59] reported that $28.1 \%$ of arthroscopically evaluated discoid lateral menisci had peripheral rim instability; therefore, the planning and preparation of surgery should include anticipation of a possible need for meniscal stabilization by suturing.

Motoric and radio frequency tools are used for meniscal reshaping. When meniscal instability is found clinically or arthroscopically, stabilization of the meniscus should be considered providing that the meniscal tissue quality allows it. We usually use an inside-out technique for meniscal suturing and use the all-inside meniscal devices for augmentation.

Type-III unstable lateral discoid menisci can be reattached to the posterior capsule and complete meniscectomy should be condemned.

Finally, despite technical improvements in arthroscopic surgery, arthroscopic procedures for treatment of discoid meniscus are considered technically difficult due to the abnormal size, height, and quality of the discoid meniscus $[28,30]$. 


\section{Treatment outcome}

Meniscectomy of the lateral meniscus harbors a detrimental effect on the lateral knee. A retrospective review of patients who underwent arthroscopic partial lateral meniscectomy for lateral meniscus tears demonstrated that early results for partial lateral meniscectomy could be quite good, but that significant deterioration of functional results and decreased activity level can be anticipated [60]. The results of total or near-total meniscectomy of non-discoid meniscus in pediatric patients are poor, with the risk of early arthrosis [52, 53].

There has been a trend toward choosing meniscal preservation procedures for treating discoid lateral meniscus. Aichroth et al. [28] reviewed 52 children with 62 discoid lateral menisci and an average follow-up of 5.5 years. The children's average age at operation was 10.5 years and the mean delay in diagnosis was 24 months. Of knees with symptomatic torn discoid menisci, 48 underwent open total lateral meniscectomy, 6 had arthroscopic partial meniscectomy, and 8 with intact discoid menisci were left alone. The reported outcomes were $37 \%$ of the knees with excellent results, $47 \%$ with good results, $16 \%$ with fair results, and no poor results. Early degenerative changes were seen in the lateral compartment in three knees of older patients (over 16 years of age) with a follow-up period of 11,13 , and 18 years after total meniscectomy. The authors concluded that arthroscopic partial meniscectomy should be recommended only when the posterior attachment of the discoid meniscus is stable and that total meniscectomy is indicated for the Wrisberg-ligament type of discoid meniscus with posterior instability.

Washington et al. [61] reported good or excellent results in 13 of 18 knees of patients with a mean age of 17 years (8-28 years). Radiographs of 9 knees ( 8 patients with a mean age of 15 years; 7-26 years) showed evidence of slight narrowing of the joint space in only 3 of them.

Raber et al. [62] reviewed the results of total meniscectomies performed in 17 knees (14 children) for discoid lateral meniscus at a mean follow-up of 19.8 years (range 12.5-26.0 years). They found that 10 of these knees had clinical symptoms of osteoarthrosis. Plain radiographs were available for 15 knees and 10 showed osteoarthritic changes.

Aglietti et al. [63] reported 10-year follow-up results of arthroscopic meniscectomies for symptomatic discoid lateral menisci in 17 adolescents. They found no correlation between the type of meniscectomy (partial or total) and the clinical and radiographic results. Development of radiographic changes, such as minor osteophytes in the lateral compartment of 8 knees and less than $50 \%$ narrowing of the lateral joint space, was found in 11 knees. The reported clinical results were excellent or good in 16 of their 17 patients.

It should be emphasized that excellent or good clinical reports after a 10-year follow-up in this age group calls for a critical appraisal. The follow-up period puts those patients in their mid-to late twenties. In the face of the reported high incidence of ominous premature radiographic arthritic changes in this and the other series, a longer follow-up period is needed.

Currently, the indications and results of treatment options, such as meniscal transplantation for knees with a history of discoid lateral meniscectomies and meniscectomy, have not yet been conclusively defined. There is some experience in performing allograft meniscal transplantation for those cases but no reports have appeared in the literature thus far. Heightened awareness of the clinician to the possibility of discoid meniscus, its variable presentations and complications, and management considerations may improve therapeutic outcome.

Acknowledgments Esther Eshkol is thanked for editorial assistance.

\section{References}

1. Young R (1889) The external semilunar cartilage as a complete disc. In: Cleland J, Mackay J, Young R (eds) Memoirs and memoranda in anatomy. Williams and Norgate, London, pp 179

2. Watson-Jones R (1930) Specimen of internal semilunar cartilage as a complete disc. Proc R Soc Med 23:588

3. Andrish J (1996) Meniscal injuries in children and adolescents: diagnosis and management. J Am Acad Orthop Surg 4:231237

4. Kaplan EB (1955) Discoid lateral meniscus of the knee joint. Bull Hosp Joint Dis 16:111-124

5. Clark C, Ogden J (1983) Development of the menisci of the human joint: morphologic changes and their potential role in childhood meniscal injury. J Bone Joint Surg Am 65:538-547

6. Jordan M (1996) Lateral meniscal variants: evaluation and treatment. J Am Acad Orthop Surg 4:191-200

7. Greis PE, Bardana DD, Holstrom MC, Burks RT (2002) Meniscal injury: basic science and evaluation. J Am Acad Orthop Surg $10: 168-176$

8. Ikeuchi H (1982) Arthroscopic treatment of the discoid lateral meniscus. Technique and long-term results. Clin Orthop Relat Res 167:19-28

9. Nathan PA, Cole SC (1969) Discoid meniscus. A clinical and pathologic study. Clin Orthop Relat Res 64:107-113

10. Jeannopoulos CL (1950) Observations on discoid menisci. J Bone Joint Surg Am 32:649-652

11. Dickason JM, Del Pizzo W, Blazina ME, Fox JM, Friedman MJ, Snyder SJ (1982) A series of ten discoid medial menisci. Clin Orthop Relat Res 168:75-79

12. Kocher MS, Micheli LJ (2001) The pediatric knee: evaluation and treatment. In: Insall JN, Scott WN (eds) Surgery of the knee. 3rd edn. Churchill-Livingstone, New York, pp 1356-1397

13. Dickhaut SC, DeLee JC (1982) The discoid lateral meniscus syndrome. J Bone Joint Surg Am 64:1068-1073

14. Jordan M, Duncan J, Bertrand S (1993) Discoid lateral meniscus: a review. South Orthop J 2:239-253 
15. Smillie I (1948) The congenital discoid meniscus. J Bone Joint Surg Br 30:671-682

16. Gebhardt MR, Rosenthal RK (1979) Bilateral lateral discoid meniscus in identical twins. J Bone Joint Surg Am 61:1110 1111

17. Dashefsky J (1971) Discoid lateral meniscus in three members of a family: case reports. J Bone Joint Surg Am 53:1208-1210

18. Watanabe M, Takada S, Ikeuchi H (1969) Atlas of Arthroscopy. Igaku-Shoin, Tokyo

19. Monllau JC, Leon A, Cugat R, Ballester J (1998) Ring-shaped lateral meniscus. Arthroscopy 14:502-504

20. Bin SI, Kim JC, Kim JM, Park SS, Han YK (2002) Correlation between type of discoid lateral menisci and tear pattern. Knee Surg Sports Traumatol Arthrosc 10:218-222

21. Shahriaree H (ed) (1992) O'Conner's Textbook of arthroscopic surgery. Lippincott, Philadelphia, pp 318-321

22. Kroiss F (1910) Die Verletzungen der Kniegelenkoszwischenknorpel und ihrer Verbindungen. Beitr Klin Chir 66:598-801

23. Woods GW, Whelan JM (1990) Discoid meniscus. Clin Sports Med 9:695-706

24. Rosenberg TD, Paulos LE, Parker RD, Harner CD, Gurley WD (1987) Discoid lateral meniscus: case report of arthroscopic attachment of a symptomatic Wrisberg-ligament type. Arthroscopy 3:277-282

25. Neuschwander DC, Drez D, Finney TP (1992) Lateral meniscal variant with absence of posterior coronary ligament. J Bone Joint Surg Am 74:1186-1190

26. Albertsson M, Gillquist S (1998) Discoid lateral meniscus: a report of 29 cases. Arthroscopy 4:211-214

27. Fleissner PR, Eilert RF (1999) Discoid lateral meniscus. Am J Knee Surg 12:125-131

28. Aichroth PM, Patel DV, Marx CI (1991) Congenital discoid lateral meniscus in children: a follow up study and evaluation of management. J Bone Joint Surg Br 73:932-939

29. Bellier G, Dupont JY, Larrain M, Caudron C, Carlioz H (1989) Lateral discoid meniscus in children. Arthroscopy 5:52-56

30. Pellacci F, Montanari G, Prosperi P, Galli G, Celli V (1992) Lateral discoid meniscus: treatment and results. Arthroscopy 8:526-530

31. Kocher MS, DiCanzio J, Zurakowski D, Micheli LJ (2001) Diagnostic performance of clinical examination and selective magnetic resonance imaging in the evaluation of intraarticular knee disorders in children and adolescents. Am J Sports Med 29:292-296

32. Harvell JJ, Fu F, Stanitski C (1989) Diagnostic arthroscopy of the knee in children and adolescents. Orthopedics 12:1555-1560

33. Haus J, Refior H (1993) The importance of arthroscopy in sports injuries in children and adolescents. Knee Surg Sports Traumatol Arthrosc 1:34-38

34. Morrissy R, Eubanks R, Park J (1982) Arthroscopy of the knee in children. Clin Orthop Relat Res 162:103-107

35. Stanitski C (1998) Correlation of arthroscopic and clinical examinations with magnetic resonance imaging findings of injured knees in children and adolescents. Am J Sports Med 26:2-6

36. Suman R, Stother I, Illingworth G (1984) Diagnostic arthroscopy of the knee in children. J Bone Joint Surg Br 66:535-537

37. Picard JJ, Constantin L (1964) Radiological aspects of the discoid meniscus. J Radiol Electrol Med Nucl 45:839-841

38. Kerr R (1986) Radiologic case study: discoid lateral meniscus. Orthopedics 8:1142-1147

39. Najafi J, Bagheri S, Lahiji FA (2006) The value of sonography with micro convex probes in diagnosing meniscal tears compared with arthroscopy. J Ultrasound Med 25:593-597

40. Achour NA, Tlili K, Souei MM, Gamaoun W, Jemni H, Dali KM, Dahmen J, Hmida RB (2006) Le menisque discoide chez l'enfant: aspects echographiques. J Radiol 87:35-40
41. Samoto N, Kozuma M, Tokuhisa T, Kobayashi K (2002) Diagnosis of discoid lateral meniscus of the knee on MR imaging. Magn Reson Imaging 20:59-64

42. Singh K, Helms CA, Jacobs MT, Higgins LD (2006) MRI appearance of Wrisberg variant of discoid lateral meniscus. AJR Am J Roentgenol 187:384-387

43. Kim YG, Ihn JC, Park SK, Kyung HS (2006) An arthroscopic analysis of lateral meniscal variants and a comparison with MRI findings. Knee Surg Sports Traumatol Arthrosc 14:20-26

44. Yoshida S, Ikata T, Takai H, Kashiwaguchi S, Katoh S, Takeda Y (1998) Osteochondritis dissecans of the femoral condyle in the growth stage. Clin Orthop Relat Res 346:162-170

45. Mitsuoka T, Shino K, Hamada M, Horibe S (1999) Osteochondritis dissecans of the lateral femoral condyle of the knee joint. Arthroscopy 15:20-26

46. Terashima T, Ohkoshi Y, Yamamoto K, Ebata W, Nagasaki S, Nishiike J, Hashimoto T, Yamane H (2005) The pathogenesis of osteochondritis dissecans in the lateral femoral condyle associated with lateral discoid meniscus injury. Paper presented at the Biennial Congress of International Society of Arthroscopy, Knee Surgery and Orthopaedic Sports Medicine (ISAKOS), Hollywood, Florida, USA, 3-7 April 2005

47. Raber DA, Friederich NF, Hefti F (1998) Discoid lateral meniscus in children. Long term follow-up after total meniscectomy. J Bone J Surg Am 80:1579-1586

48. Stanitski CL, Bee J (2004) Juvenile osteochondritis dissecans of the lateral femoral condyle after lateral discoid meniscal surgery. Am J Sports Med 32(3):797-801

49. Baratz ME, Fu FH, Mentago R (1986) Meniscal tears: the effect of menisectomy and of repair on intra-articular contact areas and stress in the human knee. Am J Sports Med 14:270-274

50. Fairbank TJ (1948) Knee joint changes after meniscectomy. J Bone Joint Surg Br 30:664-670

51. Zaman M, Leonard MA (1981) Meniscectomy in children: results in 59 knees. Injury 12:425-428

52. Manzione M, Pizzutillo PD, Peoples AB, Schweizer PA (1983) Meniscectomy in children: a long-term follow-up study. Am J Sports Med 11:111-115

53. Wroble RR, Henderson RC, Campion ER, El-Khoury GY, Albright JP (1992) Meniscectomy in children and adolescents. A long-term follow-up study. Clin Orthop Relat Res 279:180-189

54. Abdon P, Turner MS, Pettersson H, Lindstrand A, Stenström A, Swanson AJG (1990) A long-term follow-up study of total meniscectomy in children. Clin Orthop Relat Res 257:166-170

55. Hayashi LK, Yamaga H, Ida K, Miura T (1988) Arthroscopic meniscectomy for discoid lateral meniscus in children. J Bone and Joint Surg Am 70:1495-1500

56. Kim SJ, Kim DW, Min BH (1995) Discoid lateral meniscus associated with anomalous insertion of the medial meniscus. Clin Orthop Relat Res 315:234-237

57. Adachi N, Ochi M, Uchio Y, Kuriwaka M, Shinomiya R (2004) Torn discoid lateral meniscus treated using partial central meniscectomy and suture of the peripheral tear. Arthroscopy 20:536-542

58. Shiozaki Y, Horibe S, Mitsuoka T, Nakamura N, Toritsuka Y, Shino K (2002) Prediction of reparability of isolated semilunar lateral meniscus tears by magnetic resonance imaging. Knee Surg Sports Traumatol Arthrosc 10:213-217

59. Klingele KE, Kocher MS, Hresko MT, Gerbino P, Micheli LJ (2004) Discoid lateral meniscus: prevalence of peripheral rim instability. J Pediatr Orthop 24:79-82

60. Jaureguito JW, Elliot JS, Lietner T, Dixon LB, Reider B (1995) Arthroscopy 11:29-36

61. Washington ER, Root L, Liener UC (1995) Discoid lateral meniscus in children-long-term follow-up after excision. J Bone Joint Surg Am 77:1357-1361 
62. Raber DA, Friederich NF, Hefti F (1998) Discoid lateral meniscus in children: long-term follow-up after total meniscectomy. J Bone Joint Surg Am 8:1579-1586
63. Aglietti P, Bertini FA, Buzzi R, Beraldi R (1999) Arthroscopic meniscectomy for discoid lateral meniscus in children and adolescents: 10-year follow-up. Am J Knee Surg 12:83-87 